
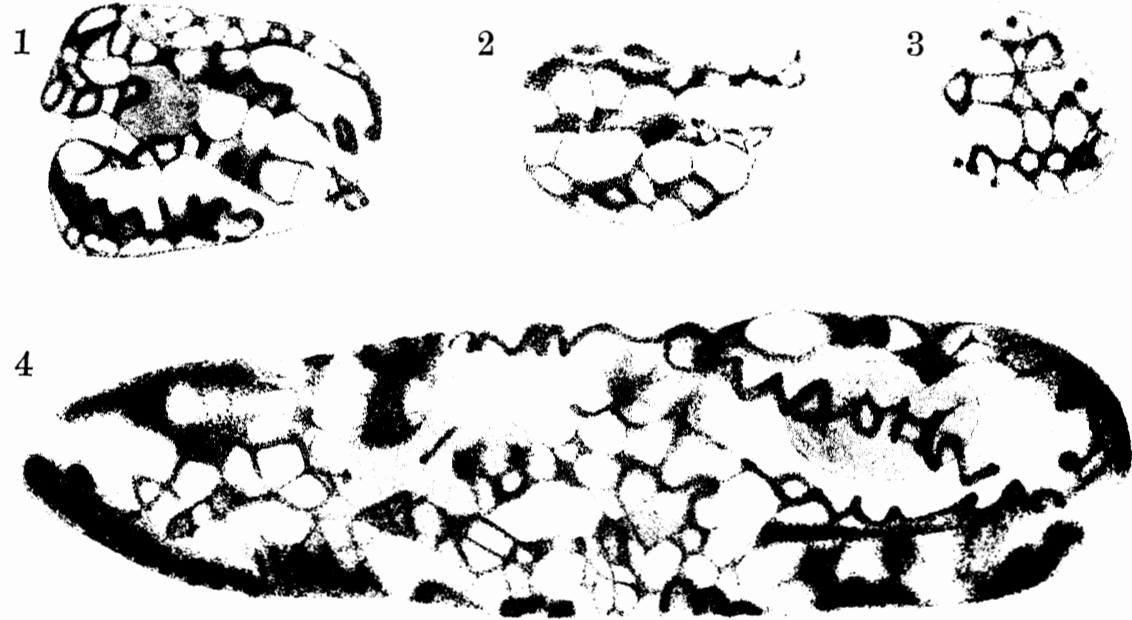

5

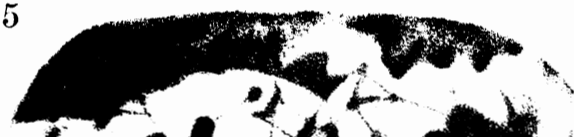
$\operatorname{lom}^{2} \cos ^{2}$

8

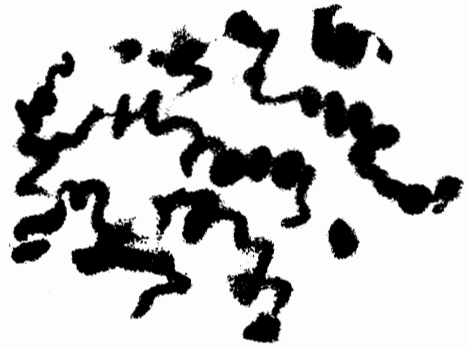

9

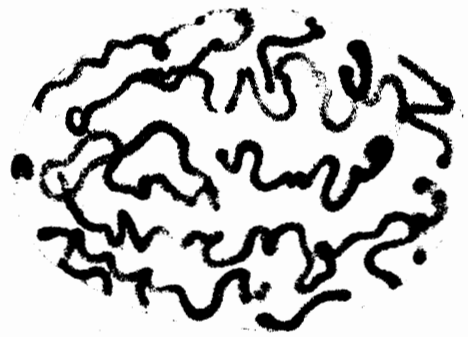

6
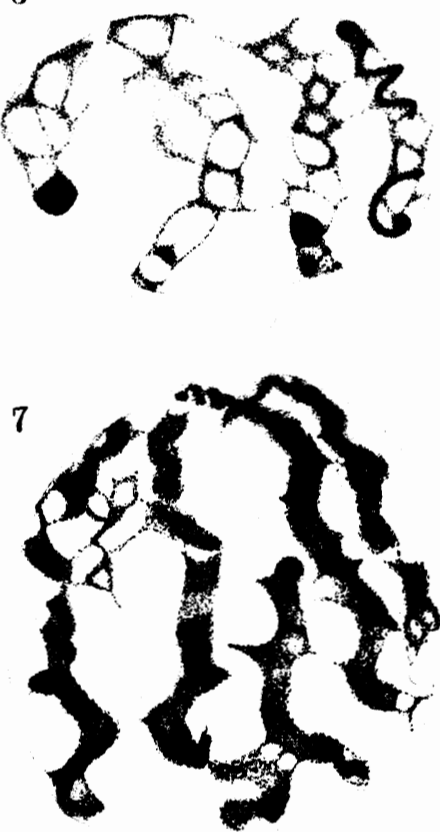

10

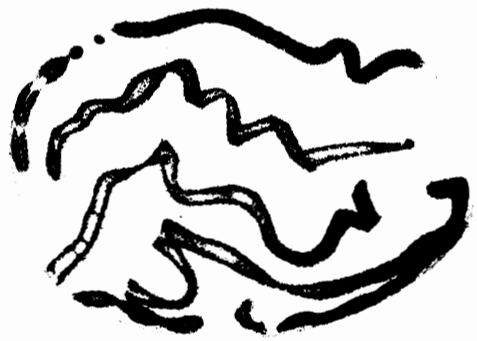




\title{
On the So-called Longitudinal Split of Chromosomes in the Telophase.
}

\author{
(Preliminary Note)
}

By

Yoshinari Kuwada.

With Plate II.

The idea is now growing that "crossing-over" occurs at an earlier stage than that assumed on the basis of JANssens's original chiasmatype theory (Plough, ${ }^{1)}$ 1917, Wilson, ${ }^{2}$ ) 1920), perhaps during the synaptic phase or slightly later. Since this idea is not in harmony with the hypothesis of telosynapsis, even in the sense of FARMER, it is most desirable from the standpoint of the chromoscmetheory of heredity, to make a study of the so-called longitudinal split of the chromosomes in the telophase, which. if it is a true split, seems to oppose the hypothesis of parasynapsis (FARMER, 1912, DigBY, ${ }^{4)}$ 1919). The present work has been undertaken at the suggestion of Professor E. B. WiLson during the writer's stay in Columbia University. The work has been, to his great regret, temporarily interrupted and in the following few pages the results, though very incomplete, are briefly mentioned so far as obtained.

There are two different opinions on the questions at what stage of the nuclear life-cycle the longitudinal splitting of chromosomes. for the next division takes place; one opinion is that it takes place in the telophase of the preceding nuclear divison, and the other that it comes to sight for the first time in the early prophase of the nucleus which is preparing for its own division. An aspect in anaphasic, or more generally in telophasic chromosomes which at first sight looks very much like a split or sign of split, has been seen in a number of

I) Jour. Exp. Zoology. Vol. 24. 1917.

2) Am. Nat. Vol. 54. 1920

3) Ann. Bot. Vol. 26. 1912

4) Ann. Bot. Vol. 33. I919. 
objects by various authors of both opinions. Thus the question now seems to be rather whether it is to be regarded as a true split or not, and not whether it is an artificial or abnormal occurrence. A fact pointed out by $\operatorname{SHARP}^{1)}$ (1914) that the light spaces are not only confined within the median region of chromosomes, but are also found near or against the periphery of chromosomes-i. e. a condition to be regarded as nothing but the results of the process of vacuolization in chromosomes-is opposed to the opinion that it is a true split. This fact, alone, however, is not very convincing, because the process of vacuolization in chromosomes approaching the resting stage must be admitted, even if we accept the anaphasic split. So we, like SHARP, would like to take another question as a critical basis for the judgement concerning the true nature of the so-called split. This is, whether or not the new chromosomes produced by this "split" retain their individuality through the resting stage and reappear in the early stage of the next prophase, retaining their double nature just as they were in the preceding telophase. Fraser and SNELL ${ }^{2)}$ (1911), and others believe that they do, but SHarp's paper of 1914 shows that these authors may have omitted some important stages. Negative results are less reliable than positive, and if we can make sure of the positive results, then the question will be naturally solved.

Having this view in mind the writer has undertaken to see whether the stages described by SHARP occur normally or not, and has obtained results in general almost the same as his. The present work has been done with root-tips of Vicia Faba, fixed with various methods and every care has been taken in regard to dehydration and infiltration of paraffin. For dehydration a series of percentages of alcohol, which consists of twelve grades, starting from $21 / 2 \%$ up to the absolute alcohol and for clearing a series of alcohol-chloroform mixture of seven grades, beginning with $2 \frac{1}{2} \%$ up to the pure chloroform, has been used. FLemming's strong solution has proved to be the best of all fixing agents generally speaking. The following brief accounts are based on preparations made from the material fixed with this solution for two different lengths of time, one for 12 hours and one for 24. For staining, Heidenhain's haematoxylin has been exclusively employed.

I) La Cellule. t. XXIX, 19r4.

2) Ann. Bot. Vol. 25. 1911. 
A light space along the median line of chromosomes is seen in every chromosome in the telophase; its structure is difficult to see in the beginning of the stage, and it seems just like a split, but later, when the vacuolization of chromosomes progresses further, it becomes clearer. The chromosomes take a form of a reticulate band consisting mostly of a row of meshes. In Fig. 2, drawn from a piece of a telophasic nucleus, three of these reticulate bands, each representing a chromosome or a part of one, are seen. The band in the upper line shows a feature resembling a split in the left half, while in the half towards the right a zigzag chromatic thread, formed by the breaking down of one side of the meshes, is visible. In the second band, can be seen nearly the same aspect as that of the left hand sicie of the first band, while the last one consists of a regular row of meshes. This preparation is made from material fixed with FLEM MING's strong solution for 12 hours. The material was not so well fixed as was that with the same solution for 24 hours. Fig. 1 is drawn from a preparation made from material fixed with FLEmming's strong solution for 24 hours. The details of the band here are more clearly shown than those in Fig. 2. The thick parts in the meshes of the band present a somewhat zigzag or spiral appearance, a feature which is very much like that in the early prophase, as we shall see later. That this nucleus is in the telophase is apparent, from the fact that in the next ccll there is a piece of a nucleus, in which chromosumes, just in the same stage as those in Fig. 1, are present.

From both sides of the band the substance becomes drawn out to form anastomoses with other chromosomes, and thus the nucleus gradually enters the resting stage. Fig. 3 and the uppermost chromosome in Fig. 1 show these processes. When the preparation for the next division begins, the anastomoses gradually disappear, and individual chromosomes become distinct again, as they were in the preceding telophase, retaining their form of a reticulate band. The mesh-work is, however, never so simple in general, as in the telophase, but much more complicated. This may be due to the fact that the anastomoses of chromatic substances had highly developed before the nucleus reached a complete resting stage. These old chromosomes mostly run parallel with each other as they were when they entered the telophase. New chromosomes begin to appear inside the old chromosomes in the form of a single, zigzag or little coiled thread, and not as a double thread. The general feature is very much like the racuolated chromosomes in the telophase (Fig. 1). In agreement 
with Sharp's paper of 1914 the writer is inclined to the view that when condensation together with a somewhat irregular break occurs in the sides of the meshes the reticulate band forms a zigzag or cciled new chromosome, though the details of the morphological relation between the new chromosomes and the meshwork remain not yet thoroughly solved. Figs. 4 and 5 show conditions of this stage. These figures are optical sections and demonstrated sharply only in the central parts of the figure. In Fig. 4 we find a reticulate band of an old chromosome running obliquely from the upper left hand side to the lower middle part of the figure, and another one running almost parallel with the former one about in the center of the figure, which shows the beginning of a new chromosome in the upper side of the band. Two other bands running parallel with these reticulate bands are found in the upper part of the right hand side of the figure, these are in a more advanced stage and the formation of new zigzag or slightly coiled chromosomes is seen very clearly, especially in the upper one, a few anastomoses remaining between the other bands. This nucleus is located in a very long cell and there can be no mistake in diagnosing its stage as an early prophase, since the long axis of the cell or of the nucleus and the direction of chromosomes run parallel to the general axis of the division figure in the napidly growing root-tip. Furthermore no nucleus in the corresponding stage is found in the surrounding cells. Fig. 5 shows almost the same stage. We find two bands in the center of the figure, the lower one being less advanced than the upper, where the formation of a new chromosome is clearly seen. In these two nuclei all the nucleoli remain quite unstained, so that the presence of the nucleoli does not obscure the details of the band.

Professor WILson has kindly given the writer the privilege of studying an excellent preparation of early prophase in a spermatogonial division of Phrynotettix, which shows quite the same feature as in Vicia. Inside an old chromosome in the form of a reticulate band, which is a morphological descendant of a chromosome in the preceding telophase (Wenrich, ${ }^{1)}$ 1916), a new spiral solid chromosome can be clearly seen (Fig. 6). It is a very interesting fact that

I) Bull. Museum Comp. Zoology Harvard Coll. Vol. LX. 1916. 
the morphology and gen csi of old and new chromosomes seem quite to coincide in the plant and the animal.

The anastomoses between, as well as inside the old chromosomes gradually disappear, and the new chromosomes, occupying the place of the old ones, and retaining their zigzag or coiled form, run distinctly free from their neighbours. Fig. 8 represents a nucleus in this stage. This may, however, be a little abnormal, in the possession of a little more of the chromatic substances, though it is not a rare occurrence. These chromosomes run mostly parallel with each other, and also, not infrequently, show a tendency to converge towards the nucleolus especially in round nuclei. Fig. 7, which presents an earlier stage than that of Fig. 8, shows very well one of the situations, although the material is not well fixed, being treated with the fixative for 12 hours, so that the figure presents a somewhat abnormal appearance from the fact that much more chromatic substances are found in the old chromosomes than in those well fixed, and the new chromosomes are too obscure to show their morphological details. The new coiled chromosomes begin to get loose, to straighten out, and to equalize. When this stage is reached, the longitudinal split of chromosomes appears to be seen for the first time in places (Fig. 9). The straighter and more equalized the chromosomes become, the more distinctly the split appears (Fig. 10). Up to these stages no sign of split has been seen with accuracy. In the stage represented by Fig. 8 whether the split begins to appear or not remains undetermined.

As will be seen by the foregoing, it seems probable that the arrangement of the essential parts of chromosomes is in a form approaching a zigzag or coil through the telophase and resting stage up to the formation of the new chromosomes in the next prophase. A light line in the axis of anaphasic chromosomes, which is interpreted by some authors to be an axial vacuolization of chromosomes or a phenomenon due to the different consistency in the axial and peripheral portion, may be interesting in connection with the feature of chromosomes in the telophase. The coiled, new chromosomes become straightened out, become longitudinally split and shorten again into the thicker and shorter definitive chromosomes, getting more chromatic substances-during the processes. We have no adequate evidence, at present, to solve the question as to how the essential parts of chromosomes are arranged in the condensed chromosomes; but there 
is some probability, that certain mechanisms exist, possibly during the process of shortening, which give such a basis to the telophasic chromosomes as to present a feature of zigzag or coiled arrangement of the essential parts. This arrangement, by condensation, develops directly into the new chromosomes, and thus the individuality of chromosomes may be maintained from generation to generation of the nuclear life-cycle.

If we admit a true split in the telophase, we must assume that the chromatic parts of an old chromosome, which form both sides of the reticulate band, represent new chromosomes. Both sides of the reticulate band will appear again in the prophase of the next nuclear life-cycle in the same way as they were in the preceding telophase. Thus they must develop further into definitive chromosomes in a paired arrangement side by side; but our studies reveal that such is not the case. New chromosomes never appear as double threads running together in a straight, zigzag or spiral course, as we should expect if we regard the split in the telophase as a true one. They are single threads taking the form of a zigzag or irregularly coiled thread. The so-called longitudinal halves of the old chromosomes separated by an assumed split in the telophase do not develop into two definitive chromosomes but taken together produce a single zigzag or coiled new chromosome. They can not, therefore, be regarded as independent daughter chromosomes; and they do not retain their individuality through the whole interkinesis from the beginning of telophase to the end of the next prophase, where the definitive chromosomes can easily be seen. There is therefore no evidence to support the view that a true longitudinal splitting of chromosomes occurs in the telophase, and no reason to oppose the hypothesis of parasynapsis by assuming the two members of the amplitenc threads to be sister chromosomes that are reuniting after a temporary separation.

The writer has the pleasant duty to offer his sincerest thanks to Professor E. B. Wilson under whose suggestion and advice the present work has been carried on, and through whose kindness all facilities have been given. The writer's thanks are also due to Professor R. A. HARPER who has very kindly given the liberty to use his laboratory during the summer vacation, and to Professor L. W. SharP in Cornell University, who has, during the writer's visit to 
him, kindly shown his excellent preparations of the root-tips of Vicia, upon which his paper of 1914 is based.

New York, Oct. I2, 1920.

\section{Explanation of the Plate II.}

All the figures are magnified to the same power; drawn with the aid of an ABBE's camera lucida, and Leitz's achromatic immersion lens $1 / 16 /$ and SPENCER's ocular Io are used for the study. Figs. I-5 and Figs. 7-1o are from root.tips of Vicia Faba and Fig. 6 from Professor WiLsos's preparation of Phrynotettix.

Fig. I. A nucleus in telophase showing vacuolization of chromosomes. Fixed for 24 hours.

Fig. 2. A piece of a nucleus in telophase. Fixed for 12 hours.

Fig. 3. The same in a little later stage. Fixed for 12 hours.

Fig. 4. A nucleus in early prophase showing development of new chromosomes. Fixed for 24 hours.

Fig. 5. The same. Fixed for 24 hours.

Fig. 6. A nucleus in early prophase of Spermatogonial division of Phrynotettix showing development of a new chromosome.

Fig. 7. A nucleus in a little later prophase, where anastomoses between chromo. somes almost disappear leaving a small remnant in the upper part of the figure. Fixed for 12 hours.

Fig. 8. A nucleus in a later stage of prophase showing spiral aspect of new chromosomes. Fixed for 24 hours.

Fig. 9. A nucleus in a much later stage of prophase showing a sign of longitudinal splitting of new chromosomes in a few places. Fixed for 24 hours.

Fig. Io. A nucleus in a much advanced stage of prophase showing longitudinal splitting of the chromosomes very clearly. Fixed for 24 hours. 